University of Nebraska - Lincoln DigitalCommons@University of Nebraska - Lincoln

U.S. Air Force Research

U.S. Department of Defense

2013

\title{
Direct flutter and limit cycle computations of highly flexible wings for efficient analysis and optimization
}

Bret Stanford

Wright-Patterson AFB

Philip Beran

Wright-Patterson AFB

Follow this and additional works at: http:// digitalcommons.unl.edu/usafresearch

Part of the Aerospace Engineering Commons, and the Aviation Commons

Stanford, Bret and Beran, Philip, "Direct flutter and limit cycle computations of highly flexible wings for efficient analysis and optimization" (2013). U.S. Air Force Research. 78.

http://digitalcommons.unl.edu/usafresearch/78

This Article is brought to you for free and open access by the U.S. Department of Defense at DigitalCommons@University of Nebraska - Lincoln. It has been accepted for inclusion in U.S. Air Force Research by an authorized administrator of DigitalCommons@University of Nebraska - Lincoln. 


\title{
Direct flutter and limit cycle computations of highly flexible wings for efficient analysis and optimization
}

\author{
Bret Stanford*, Philip Beran \\ U.S. Air Force Research Laboratory, Wright-Patterson AFB, OH 45433, USA
}

\section{A R T I C L E I N F O}

\section{Article history:}

Received 17 June 2011

Accepted 5 August 2012

Available online 12 October 2012

\begin{abstract}
A B S T R A C T
The usefulness of flutter as a design metric is diluted for wings with destabilizing (softening) nonlinearities, as a stable high-amplitude limit cycle (subcritical) may exist for flight speeds well below the flutter point. It is thus desired to design aeroelastic structures such that the post-flutter behavior is as benign (i.e., supercritical) as possible, among the other constraints commonly considered in the optimization process. In order to account for these metrics in an accurate and efficient manner, direct tools are utilized to first locate the Hopf-point (flutter speed), and then to obtain a nonlinear perturbation solution via the method of multiple scales. The latter scheme provides a scalar variable whose sign and magnitude dictate the nature of the limit cycle. The accuracy of these methods is demonstrated with a high-aspect-ratio highly flexible wing, modeled with nonlinear beam finite elements and the ONERA dynamic stall tool. Stiffness and inertial design variables are allowed to vary spatially throughout the wing, in order to conduct gradient-based optimization of the limit cycle under flutter and mass constraints. The resulting wing structure demonstrates strongly supercritical behavior, as well as several design conflicts between linear (flutter) and nonlinear (limit cycles) sensitivities, which are not present in the uniform baseline wing.
\end{abstract}

Published by Elsevier Ltd.

\section{Introduction}

Design and optimization of an aeroelastic wing structure may be undertaken in order to obtain a low-mass design that satisfies several different types of constraints. These constraints may require a limitation on the peak stresses, displacements, or natural frequencies, a certain degree of control surface effectiveness, or a prescribed lower bound on the flutter speed (Haftka, 1986). Flutter, which is of primary concern here, occurs at a Hopf-bifurcation point as the flight speed is increased, a pair of complex conjugate eigenvalues of the system Jacobian cross the imaginary axis. The system is then dynamically unstable, and disturbances may quickly grow unbounded (Bisplinghoff et al., 1955). The presence of nonlinear mechanisms within the aeroelastic system may attenuate this growth, (Dowell et al., 2003) however, and produce a self-sustained limit cycle oscillation (LCO). Two scenarios may occur, as seen in Fig. 1.

If the nonlinearities are stabilizing (hardening), supercritical limit cycles can occur, resulting in (potentially) benign continuous growth as the flight speed $U$ is increased above the flutter speed. This behavior is reversible in the sense that decreasing $U$ will return the aeroelastic dynamics along the same path, and oscillations will again decay if the speed is below flutter. Contrastingly, if the nonlinearities are de-stabilizing (softening) a subcritical limit cycle exists. This limit

\footnotetext{
* Corresponding author.

E-mail address: bretkennedystanford@gmail.com (B. Stanford).
} 


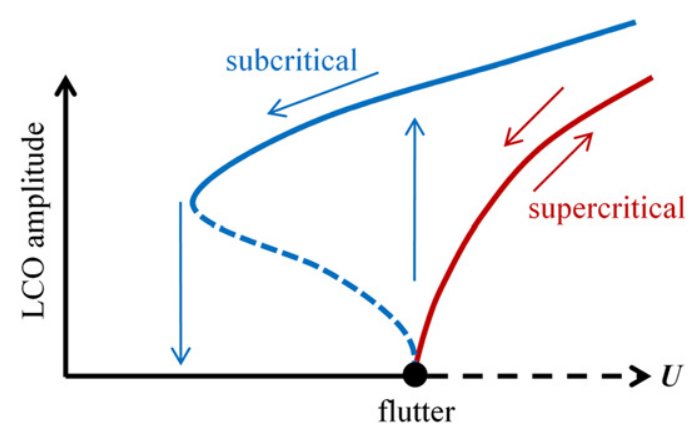

Fig. 1. Limit cycle responses emerging from a flutter point.

cycle is unstable (denoted by a dashed line in Fig. 1), and exists for speeds below the flutter speed. Commonly, higher-order stabilizing nonlinearities exist within the system (Strogatz, 1994), and the subcritical branch transitions into a stable large amplitude branch. For this scenario, as the speed is increased above the flutter point, the system will jump to this largeamplitude branch, suddenly incurring dangerously large stresses and wing deflections. Subsequently decreasing the speed below the flutter point does not immediately return the system dynamics to the origin, resulting in a hysteresis loop.

Clearly, subcritical limit cycle oscillations are highly un-desirable from a design/optimization perspective, due to both the post-flutter jump to the large-amplitude branch as well as the hysteretic behavior. Furthermore, the presence of subcritical behavior dilutes the importance of flutter speed as a constraint in the aeroelastic optimization process. Even at flight speeds below flutter, large disturbances (due to a gust, for example) may push the system dynamics onto the stable large-amplitude limit cycle branch (Patil et al., 2001). Depending upon the strength of the leading-order softening nonlinearities, this branch (and thus the threat of entrainment into a strong LCO) may exist well below the flutter speed constraint. Short of using optimization to push the flutter speed well outside the flight envelope (which may result in over conservative and heavy structures) a low-amplitude supercritical limit cycle is an attractive alternative. Wing structures may not be explicitly designed to operate within this limit cycle, but strong uncertainties in the aeroelastic behavior (Beran et al., 2006; Thomas et al., 2006) require that the nonlinear post-flutter dynamics, if encountered, must be benign.

The above discussion would indicate that aeroelastic systems prone to subcritical behavior should include limit cycle oscillation constraints during the optimization process. Such examples are rare in the literature, see Palaniappan et al. (2006), Missoum et al. (2010), and Stanford and Beran (2012). One reason for this scarcity is that limit cycle computations are very expensive, and thus not amenable to the repeated function evaluations and sensitivity analysis required for gradient-based optimization. If standard time-marching methods are utilized, many time steps are required to first ascertain the flutter boundary (beyond which oscillations do not decay), and then quantify the limit cycle behavior. The system damping at flutter is zero (Bisplinghoff et al., 1955), and so dynamics in this vicinity develop very slowly. Monolithic-time schemes (via harmonic balance (Thomas et al., 2004) or spectral elements (Stanford and Beran, 2012), for example) bypass this problem by computing only time-periodic orbits, but several computational issues exist. First, the system of equations is very large (the product of the spatial and the temporal degrees of freedom), a good initial guess (typically obtained from time-marching) is required, and convergence problems have been noted for strong nonlinearities (Stanford et al., 2010).

For an effective optimization tool, flutter and the concomitant limit cycle oscillation should be obtained efficiently, precisely and directly (i.e., as an outcome of a numerical procedure). Towards this end, direct flutter tools, which establish a nonlinear set of algebraic equations for the flutter point based upon general information about Hopf-point solutions (Griewank and Reddien, 1983) (a small oscillatory perturbation about a steady state equilibrium), were developed by Morton and Beran (1999) and extended by Badcock and Woodgate (2010), and Badcock et al. (2004). Gradients of the directly computed flutter speed with respect to a large number of design variables were obtained by Stanford and Beran (2011) for aeroelastic optimization.

Direct location of the flutter speed can then be followed by a nonlinear perturbation about this point, with the assumption of weak nonlinearity, in order to obtain an approximation for the limit cycle dynamics in the vicinity of the Hopf-point. Many computational varieties exist; the scheme used here is the well-known method of multiple scales (MMS), described in many texts (e.g., Nayfeh and Balachandran, 1995). A perturbation solution is built upon a slow and a fast time scale, which are treated as independent variables; the additional variables can be used to remove unwanted secular terms (Strogatz, 1994). The resulting computations reduce the system dynamics to a two-degree-of-freedom system (which represent the motions of the critical aeroelastic mode shape). The nonlinear dynamics are thus distilled down to a few directly computed parameters which provide information on whether the LCO is supercritical or subcritical (Fig. 1), and the severity of the branch. Both direct computations, flutter and nonlinear perturbation, are obtained in an inexpensive manner.

Information pertaining to high-amplitude, strongly nonlinear dynamics (far away from the flutter speed) is unreliable from a perturbation method. The local behavior predicted by MMS, however, will be sufficient to optimize wing structures as described above, converting dangerous subcritical behavior into supercritical LCOs. Multiple-scale analysis of aeroelastic 
systems can be found in Chandiramani et al. (1996), Beran (1999), Ghommem et al. (2010), Gilliatt et al. (2003), Paolone et al. (2006), and Nayfeh et al. (2012), and a similar reduction of the limit cycle behavior to a few critical parameters is conducted by Librescu et al. (2002) (the Lyapunov first quantity) and Woodgate and Badcock (2007) (center manifold theory). To the best of the authors' knowledge, it has not been used for design optimization, aero-structural or otherwise.

As documented by Dowell et al. (2003), the physical source of the nonlinearity within the aeroelastic limit cycle may be structural (geometric stress stiffening, buckling, freeplay, damping), aerodynamic (shock motions, flow separation), or due to the coupling between the two. Multiple (and potentially conflicting) nonlinearities may be simultaneously present, which may cause the subcritical behavior seen in Fig. 1, where an unstable branch transitions into a stable one. The current work is concerned with the aeroelasticity of highly flexible wings of large aspect ratio. Such wings are found in highaltitude long-endurance (HALE) aircraft, a class of solar-powered unmanned air vehicles.

Aeroelastic modeling of these systems has garnered a great deal of attention in recent years, due to both the complex nonlinear unsteady coupled physics of the problem, as well as the relevancy to non-traditional HALE aircraft. Most researchers build models of highly flexible wings by coupling nonlinear beam models to strip theory aerodynamics (Nayfeh et al., 2012; Nichkawde et al., 2006; Patil et al., 2001; Shearer and Cesnik, 2007; Tang and Dowell, 2001, 2002) or panel methods (Demasi and Livne, 2009; Palacios et al., 2010; Patil and Hodges, 2004; Wang et al., 2010). The former option is utilized here; both subcritical and supercritical LCOs have been observed, and are "dependent on a delicate balance between stall aerodynamics and the structural nonlinear forces" (Tang and Dowell, 2001). The direct tools described above should be able to elucidate the physics that dictate this balance, as well as methods by which the wing structure/inertia can be tailored to obtain benign post-flutter dynamics.

\section{Aeroelastic modeling framework}

The highly flexible wing model studied here (Fig. 2) is identical to that considered by Tang and Dowell (2001, 2002), due in large part to the availability of experimental flutter and limit cycle wind tunnel data, as well as the author's success in correlating aeroelastic models to this data. The length of the wing (root to tip) is $0.4508 \mathrm{~m}$, the chord is $0.0508 \mathrm{~m}$, and a NACA 0012 airfoil is used. A flat steel spar $(1.27 \mathrm{~cm}$ by $0.127 \mathrm{~cm})$ runs through the middle of the wing, providing the bulk of the stiffness. A tip body (an aluminum bar $10.16 \mathrm{~cm}$ long and $0.95 \mathrm{~cm}$ in diameter) is attached to the wingtip in order to reduce the torsional natural frequency of the system and to obtain flutter speeds within the range of the wind tunnel. Thin flanges are also distributed along the steel spar to further lower torsional stiffness. For the complete wing, the flapwise stiffness $\left(E I_{1}\right)$ is $0.42 \mathrm{~N} \mathrm{~m}^{2}$, the edgewise stiffness $\left(E I_{2}\right)$ is $18.44 \mathrm{~N} \mathrm{~m}^{2}$, the torsional rigidity $(G J)$ is $0.95 \mathrm{~N} \mathrm{~m}^{2}$, the mass per unit length $(m)$ is $0.235 \mathrm{~kg} / \mathrm{m}$, and the rotational inertia per unit length $\left(I_{o}\right)$ is $0.205 \times 10^{-4} \mathrm{~kg} \mathrm{~m}$. Further model parameters are provided by Tang and Dowell (2001).

The aeroelastic equations of motion are briefly reviewed below. The Hodges-Dowell equations (Hodges and Dowell, 1974) are used to compute the elastic motion of the wing:

$$
\begin{aligned}
& m \cdot \ddot{v}+E I_{2} \cdot v_{, x x x x}+\left(E I_{2}-E I_{1}\right) \cdot\left(\phi \cdot w_{, x x}\right)_{, x x}=R_{v}, \\
& m \cdot \ddot{w}+E I_{1} \cdot w_{, x x x x}+\left(E I_{2}-E I_{1}\right) \cdot\left(\phi \cdot v_{, x x}\right)_{, x x}=R_{w}, \\
& I_{o} \cdot \ddot{\phi}-G J \cdot \phi,_{x x}+\left(E I_{2}-E I_{1}\right) \cdot w_{, x x} \cdot v_{, x x}=R_{\phi},
\end{aligned}
$$

where $v, w$, and $\phi$ are the edgewise, flapwise, and torsional motions of the beam, respectively, and the right-hand-sides $R_{v}$, $R_{w}$, and $R_{\phi}$ are forces and moments associated with structural damping, gravitational loading, inertial loading of the tip body, and aerodynamic loading, respectively. It can be seen that the nonlinearity is solely due to the stiffness ratio $E I_{2} / E I_{1}$. If this ratio is unity (an isotropic beam with a square cross-section, for example), then the equations become linear. Eqs. (1)-(3) contain leading-order nonlinearities; for large deflections the accuracy will be lower than geometrically exact approaches (Patil et al., 2001, for example).

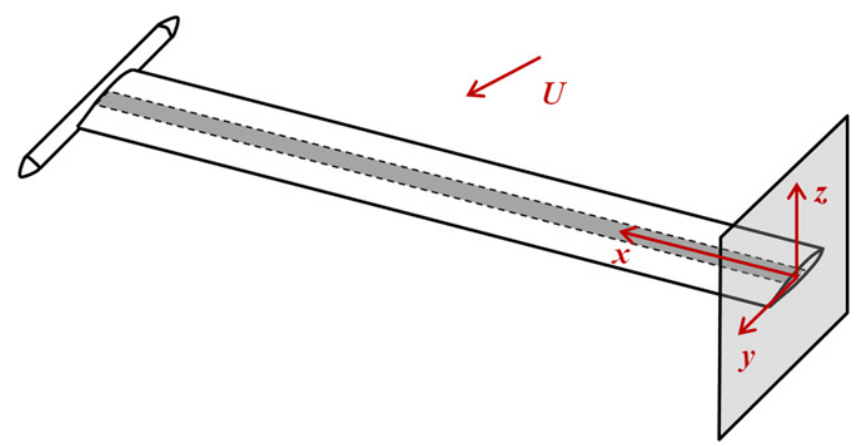

Fig. 2. High-aspect-ratio wing model studied by Tang and Dowell (2001). 
The beam of Fig. 2 is discretized into two-dimensional elements, and Eqs. (1)-(3) are converted into a system of finite elements following the usual methods (Cook et al., 2002)

$$
\boldsymbol{M} \cdot \ddot{\boldsymbol{u}}+\boldsymbol{C} \cdot \dot{\boldsymbol{u}}+\boldsymbol{F}^{\mathrm{int}}(\boldsymbol{u})=\boldsymbol{F}^{\mathrm{ext}} .
$$

The solution vector $\boldsymbol{u}$ is composed of $\left\{\begin{array}{lllll}v & w & \phi & w, x & v, x\end{array}\right\}^{T}$ at each node, $\boldsymbol{M}$ is a consistent mass matrix (which includes the effect of the tip body), $\boldsymbol{C}$ is a structural damping matrix, and $\boldsymbol{F}^{\text {ext }}$ is a force vector which includes gravitational and aerodynamic loading. The internal elastic forces are given by $\boldsymbol{F}^{\text {int }}$, which is a nonlinear function of the beam deflection $\boldsymbol{u}$. The solution is approximated as a linear combination of a relatively small number of modes $\boldsymbol{u}=\boldsymbol{\Phi} \cdot \boldsymbol{\eta}$. The modal matrix $\boldsymbol{\Phi}$ contains natural vibration modes in each column, and $\boldsymbol{\eta}$ is a vector of modal amplitudes. Inserting this approximation into the equations of motion, and pre-multiplying by $\boldsymbol{\Phi}^{T}$ provides

$$
\begin{aligned}
& \boldsymbol{\Phi}^{\mathrm{T}} \cdot \boldsymbol{M} \cdot \boldsymbol{\Phi} \cdot \ddot{\boldsymbol{\eta}}+\boldsymbol{\Phi}^{\mathrm{T}} \cdot \boldsymbol{C} \cdot \boldsymbol{\Phi} \cdot \dot{\boldsymbol{\eta}}+\boldsymbol{\Phi}^{\mathrm{T}} \cdot \boldsymbol{F}^{\mathrm{int}}(\boldsymbol{\Phi} \cdot \boldsymbol{\eta})=\boldsymbol{\Phi}^{\mathrm{T}} \cdot \boldsymbol{F}^{\mathrm{ext}}, \\
& \boldsymbol{M}_{r} \cdot \ddot{\boldsymbol{\eta}}+\boldsymbol{C}_{r} \cdot \dot{\boldsymbol{\eta}}+\boldsymbol{F}_{r}^{\mathrm{int}}(\boldsymbol{\eta})=\boldsymbol{F}_{r}^{\mathrm{ext}},
\end{aligned}
$$

where subscript $\boldsymbol{r}$ implies a reduced quantity, and the mode shapes are scaled such that $\boldsymbol{M}_{r}$ is the identity matrix. No attempt is made to write the nonlinear term $\boldsymbol{F}_{r}^{\text {int }}$ as a direct function of $\boldsymbol{\eta}$. Instead, the current value of $\boldsymbol{\eta}$ is expanded to $\boldsymbol{u}$ (via the modal matrix), $\boldsymbol{F}^{\text {int }}(\boldsymbol{u})$ is computed in the full-order space, and then compressed back down to $\boldsymbol{F}_{r}^{\text {int }}$. This may be thought of as the "on-line" approach to model reduction (Stanford et al., 2010).

The angle of attack at the wing root is $\alpha_{o}$, and the local angle of each wing section is given by Tang and Dowell (2001)

$$
\alpha=\alpha_{0}+\phi-\dot{w} /\left(U+\dot{v}+\dot{w} \cdot \alpha_{0}\right) \text {. }
$$

The ONERA stall model (developed by Tran and Petot, 1981, further discussed by Peters, 1985; Dunn and Dugundji, 1992; Tang and Dowell, 2002) can then be used to compute the unsteady aerodynamics at each section. The sectional coefficient is given as

$$
C_{z}=\tau \cdot s_{z} \cdot \dot{\alpha}+\tau^{2} \cdot k_{v z} \cdot \ddot{\phi}+C_{z 1}+C_{z 2},
$$

where $\tau=c /(2 \cdot U)$ is the non-dimensional time constant, and $C_{z}$ may be the lift or the moment coefficient. The first two terms in Eq. (8) are non-circulatory terms, and $C_{z 1}$ and $C_{z 2}$ are the linear (circulatory) and nonlinear (stall) unsteady aerodynamic terms, respectively

$$
\begin{aligned}
& \tau \cdot \dot{C}_{z 1}+\lambda_{z} \cdot C_{z 1}=\lambda_{z} \cdot\left(a_{o z} \cdot \alpha+\tau \cdot \sigma_{z} \cdot \dot{\phi}\right)+\alpha_{z} \cdot\left(\tau \cdot a_{o z} \cdot \dot{\alpha}+\tau^{2} \cdot \sigma_{z} \cdot \ddot{\phi}\right), \\
& \tau^{2} \cdot \ddot{C}_{z 2}+a \cdot \tau \cdot \dot{C}_{z 2}+r \cdot C_{z 2}=-r \cdot\left(\Delta C_{z}+\tau \cdot e \cdot \Delta C_{z \alpha} \cdot \dot{\alpha}\right) .
\end{aligned}
$$

Eq. (9) is a state-space representation of Theodorsen's function, and Eq. (10) is a second-order system governing the dynamic stall. This equation is driven by the deviation term $\Delta C_{z}$, which is the discrepancy between the specified nonlinear force curve and the linear curve (typically $a_{o z} \cdot \alpha$ ), defined as positive for a decrease in the force (Peters, 1985). Two stall curves are frequently seen in the literature: piecewise-linear (Dunn and Dugundji, 1992; Patil et al., 2001; Tang and Dowell, 2001) and cubic (Ghommem et al., 2010; Nayfeh et al., 2012; Nichkawde et al., 2006). Both are utilized here (Fig. 3 ) in order to assess their impact on the nonlinear dynamics. For the piecewise-linear curve $\Delta C_{z}$ is zero until $\alpha$ increases above $10^{\circ}$, whereas $\Delta C_{z}$ is a smooth non-zero function for small angles in the cubic model. The ONERA coefficients used in Eqs. (8)-(10) are taken from Dunn and Dugundji (1992).

The above equations are solved at each wing section, which are spaced so as to align with the finite element nodes. Having computed $C_{L}$ and $C_{M}$, the drag coefficient is obtained from an assumed quadratic $C_{D}-\alpha$ profile, and the sectional

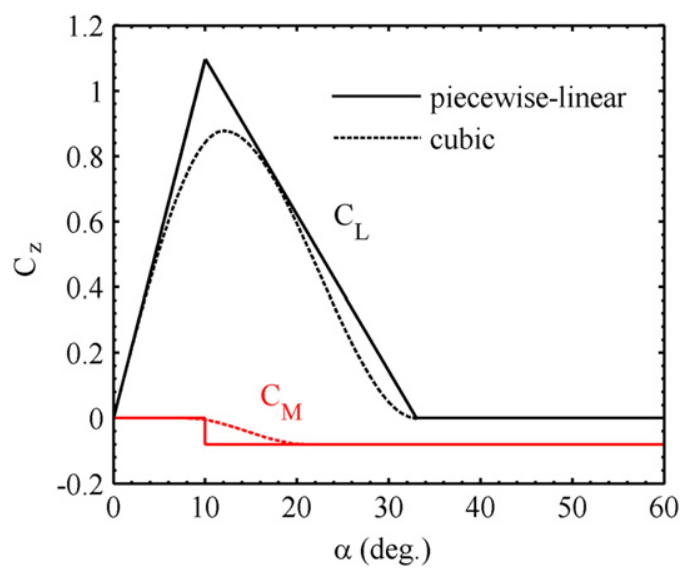

Fig. 3. Piecewise linear and cubic stall models. 
forces and moments can be computed. These nodal quantities are assumed to vary linearly over each beam element, workconsistent nodal loads are obtained (Cook et al., 2002), and finally assembled to compute the force vector $\boldsymbol{F}^{\text {ext }}$ of Eq. (4). The final set of aeroelastic unknowns is

$$
\boldsymbol{q}=\left\{\begin{array}{llllllll}
\boldsymbol{\eta} & \dot{\boldsymbol{\eta}} & \boldsymbol{C}_{L 1} & \boldsymbol{C}_{L 2} & \dot{\boldsymbol{C}}_{L 2} & \boldsymbol{C}_{M 1} & \boldsymbol{C}_{M 2} & \dot{\boldsymbol{C}}_{M 2}
\end{array}\right\},
$$

where $\boldsymbol{\eta}$ are the modal amplitudes, and $\boldsymbol{C}_{L 1}$, for example, is a vector containing $C_{L 1}$ at each wing node. For a system with $N$ finite element nodes and $N_{r}$ vibration modes, the number of aeroelastic unknowns $N_{q}=2 \cdot N_{r}+6 \cdot N$. Eqs. (6)-(10) are combined into standard first-order form

$$
\dot{\boldsymbol{q}}=\boldsymbol{R}(\boldsymbol{q}), \quad \boldsymbol{J}=\partial R / \partial \mathbf{q},
$$

where $\boldsymbol{R}$ is the residual vector (a nonlinear function of $\boldsymbol{q}$ ) and $\boldsymbol{J}$ is the analytically computed Jacobian of the system.

Results of this aeroelastic model are compared with numerical and experimental data of Tang and Dowell (2001) in Fig. 4. The left side of this figure shows the flutter velocity for different root angles $\alpha_{o}$. Following standard practices, the equilibrium solution $\boldsymbol{q}_{e}$ is first computed for which $\boldsymbol{R}\left(\boldsymbol{q}_{e}\right)=0$, the problem is linearized about this point by computing $\boldsymbol{J}$, and flutter occurs when a pair of eigenvalues of $\boldsymbol{J}$ cross the imaginary axis with increasing flow velocity $U$. At $1^{\circ}$ the equilibrium solution $\boldsymbol{q}_{e}$ is nearly zero: the gravitational load (self-weight) cancels out with the static aerodynamic load. The flutter speed is minimum at this angle, but higher for other angles due to the static deflection (negative bending for $\alpha_{o}<1^{\circ}$ and positive for angles above $1^{\circ}$ ) which nonlinearly stiffens the beam.

The right side of Fig. 4 shows limit cycle behavior for a fixed $\alpha_{o}$ of $1^{\circ}$, using the piecewise-linear stall model of Fig. 3 . The RMS amplitude of the flapwise deflection at the midpoint of the wing is plotted as a function of the flight speed $U$. The critical aeroelastic mode shape at flutter for this case is a combination of the first torsion mode and the second flapwise bending mode (Fig. 7), so midpoint deflections will be prevalent. Limit cycle behavior is computed with timeintegration (the Crank-Nicolson method with an alpha-scheme for higher-order dissipation (Cornwell and Malkus, 1992), and bears many similarities with the subcritical branch seen in Fig. 1. Once the flutter speed (35 m/s for the current model) is bypassed, the system dynamics suddenly jump to the high-amplitude stable branch. Vibrations only subsist when the flow velocity is decreased $\sim 2 \mathrm{~m} / \mathrm{s}$ below the flutter point. Obviously, the unstable branch of the LCO directly emanating from the Hopf-point is unavailable from either time marching or the experimental data.

Comparisons between the current numerical model, the numerical model of Tang and Dowell (2001), and the wind tunnel data of the same work, are satisfactory in terms of both flutter speeds and limit cycles. Differences between the numerical predictions may be largely due to the fact that the aerodynamic effect of the slender tip body is ignored for the current work, as well as the current use of discretized structural finite elements rather than the analytical modal approach of Tang and Dowell (2001).

\section{Direct methods}

As an alternative to the time marching schemes presented above, the first step of the direct methodology envisioned here is location of the Hopf-point, or flutter speed. At this point, a solution is assumed of the form

$$
\boldsymbol{q}=\boldsymbol{q}_{e}+\varepsilon \cdot \boldsymbol{P} \cdot e^{\mathrm{i} \cdot \omega \cdot t},
$$

where $\boldsymbol{q}_{e}$ is the equilibrium solution (static-aeroelastic solution, described above), $\varepsilon$ is a vanishingly small parameter, and $\boldsymbol{P}$ is the complex eigenvector associated with the critical eigenvalue $i \cdot \omega$. For the remainder of this work it will be assumed that $\boldsymbol{q}_{e}$ is the trivial solution, which becomes exactly true if gravitational effects (self-weight) are removed from the system
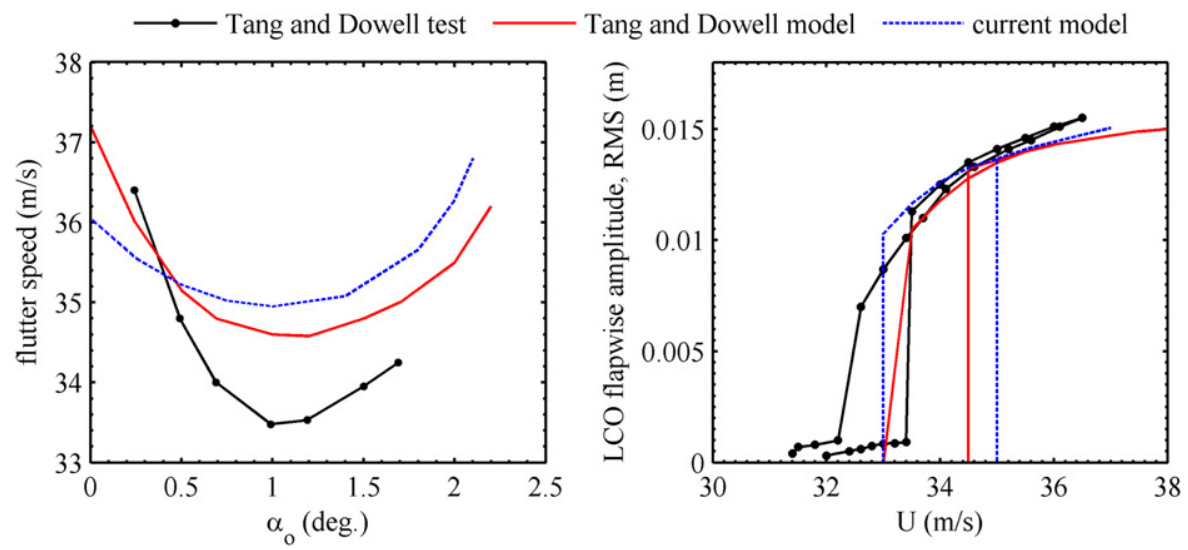

Fig. 4. Comparisons with Tang and Dowell (2001), flutter speed for different root angles of attack (left) and RMS flapwise amplitude at the midspan of the beam during a limit cycle at $\alpha_{o}=1^{\circ}$. 
of equations, and the root angle $\alpha_{o}$ is fixed at $0^{\circ}$. This assumption indicates that $d \boldsymbol{R} / d U$ is zero as well at the Hopf-point.

Inserting Eq. (13) into the equations of motion (Eq. (12)), and expanding $\boldsymbol{R}(\boldsymbol{q})$ in a Taylor series approximation, leads to the eigenproblem $\boldsymbol{J} \cdot \boldsymbol{P}=i \cdot \omega \cdot \boldsymbol{P}$. The eigenvector is uniquely defined by the condition $\boldsymbol{s}^{T} \cdot \boldsymbol{P}=i$, where $s$ is a real constant array (Griewank and Reddien, 1983). The Hopf-point is located by solving the combined system of nonlinear equations

$$
\boldsymbol{R}_{H B}(\boldsymbol{Y})=\left\{\frac{J \cdot P-i \cdot \omega \cdot \boldsymbol{P}}{\boldsymbol{s}^{T} \cdot \boldsymbol{P}-i}\right\}=0, \quad \boldsymbol{Y}\left\{\begin{array}{c}
\boldsymbol{P} \\
U \\
\omega
\end{array}\right\} .
$$

Dividing the equations of $\boldsymbol{R}_{H B}$ into real and imaginary parts leads to four equations for four unknown quantities: the real and imaginary portions of $\boldsymbol{P}$, the flutter speed, and the flutter frequency. Eq. (14) is solved with Newton iterations, with the Jacobian $\partial \boldsymbol{R}_{H B} / \partial \boldsymbol{Y}$ computed analytically. The only term needed for this procedure that is not already known is the derivative $\partial(\boldsymbol{J} \cdot \boldsymbol{P}) / \partial U$.

Experience has shown that a good initial guess $\mathbf{Y}^{0}$ is needed to prevent divergence (Morton and Beran, 1999) (or convergence to a higher critical speed, or to zero speed, which is also a Hopf-bifurcation point). As such, an eigenvaluetracking method is used, with a relatively large stepping value of $\Delta U$, to obtain a rough estimate for $\boldsymbol{Y}^{0}$. Then, the direct method (Eq. 14) is used to locate the precise flutter point (typically converging to machine accuracy within five iterations), at which point $U \rightarrow U^{*}$ and $\omega \rightarrow \omega^{*}$ (flutter speed and frequency, respectively).

Having located the flutter point, the method of multiple scales is briefly summarized next. No attempt is made here to provide a detailed derivation of the technique; the interested reader is referred to Nayfeh and Balachandran (1995) and Beran (1999) for this information.

Perturbations about the Hopf-point are defined as

$$
\boldsymbol{q}=\boldsymbol{q}_{e}^{*}+\varepsilon \cdot \hat{\boldsymbol{q}} \quad U=U^{*}+\varepsilon^{2} \cdot \hat{U}
$$

where $\boldsymbol{q}_{e}^{*}$ is the equilibrium solution at the flutter point, which as discussed above, is assumed to be zero. Substituting this perturbation into the equations of motion (Eq. 12), and expanding via a Taylor series provides (under the condition that $d \boldsymbol{R} / d U$ is zero)

$$
\dot{\hat{\boldsymbol{q}}}=\boldsymbol{J} \cdot \hat{\boldsymbol{q}}+\varepsilon^{2} \cdot \hat{U} \cdot(\partial \boldsymbol{J} / \partial U) \cdot \hat{\boldsymbol{q}}+\varepsilon \cdot \boldsymbol{C}(\hat{\boldsymbol{q}}, \hat{\boldsymbol{q}})+\varepsilon^{2} \cdot \boldsymbol{D}(\hat{\boldsymbol{q}}, \hat{\boldsymbol{q}}, \hat{\boldsymbol{q}})+\cdots,
$$

where $\boldsymbol{C}(\hat{\boldsymbol{q}}, \hat{\boldsymbol{q}})$ and $\boldsymbol{D}(\hat{\boldsymbol{q}}, \hat{\boldsymbol{q}}, \hat{\boldsymbol{q}})$ are vector-valued symmetric bilinear and trilinear directional derivative operators. These may be easily computed with finite differences. For example

$$
\boldsymbol{C}\left(\boldsymbol{W}_{1}, \boldsymbol{W}_{2}\right) \approx\left(\boldsymbol{J}\left(\boldsymbol{q}_{e}^{*}+\varepsilon_{C} \cdot \boldsymbol{W}_{1}, U^{*}\right)-\boldsymbol{J}\left(\boldsymbol{q}_{e}^{*}-\varepsilon_{C} \cdot \boldsymbol{W}_{1}, U^{*}\right)\right) \cdot \boldsymbol{W}_{2} / 4 / \varepsilon_{C}
$$

The appropriate size of $\varepsilon_{c}$ (and $\varepsilon_{D}$ ) is arrived at empirically (Beran 1999).

The local solution is expanded as

$$
\hat{\boldsymbol{q}}=\hat{\mathbf{q}}_{1}\left(T_{0}, T_{2}\right)+\varepsilon \cdot \hat{\mathbf{q}}_{2}\left(T_{0}, T_{2}\right)+\varepsilon^{2} \cdot \hat{\mathbf{q}}_{3}\left(T_{0}, T_{2}\right)+\cdots .
$$

The multiple time scales are given as $T_{o}=t$ and $T_{2}=\varepsilon^{2} \cdot t$. Substituting this expression into the expansion of Eq. (16), and equating like powers of $\varepsilon$ provides the following results. For like powers of $\varepsilon^{0}$, the solution is

$$
O(1): \hat{\mathbf{q}}_{1}=A \cdot \boldsymbol{P} \cdot e^{i \cdot \omega^{*} \cdot T_{o}}+\bar{A} \cdot \overline{\boldsymbol{P}} \cdot e^{-i \cdot \omega^{*} \cdot T_{o}} .
$$

Substituting this expression into the equation for like powers of $\varepsilon$, provides the following solution

$$
O(\varepsilon): \quad \hat{\mathbf{q}}_{2}=2 \cdot \boldsymbol{Z}_{o} \cdot A \cdot \bar{A}+2 \cdot \boldsymbol{Z}_{2} \cdot A^{2} \cdot e^{2 \cdot i \cdot \omega^{*} \cdot T_{o}}+c c,
$$

where $c c$ refers to a complex conjugate, and $\boldsymbol{Z}_{o}$ and $\boldsymbol{Z}_{2}$ are computed by solving the following systems

$$
\boldsymbol{J} \cdot \boldsymbol{Z}_{o}=-\boldsymbol{C}(\boldsymbol{P}, \overline{\boldsymbol{P}}) / 2 \quad\left(2 \cdot i \cdot \omega^{*} \cdot \boldsymbol{I}-\boldsymbol{J}\right) \cdot \boldsymbol{Z}_{2}=\boldsymbol{C}(\boldsymbol{P}, \boldsymbol{P}) / 2,
$$

where $\boldsymbol{I}$ is the identity matrix. Finally, the expressions for $\hat{\mathbf{q}}_{1}$ (Eq. 19) and $\hat{\mathbf{q}}_{2}$ (Eq. 20) are substituted into the equation for like powers of $\varepsilon^{2}$. Rather than explicitly solving for $\hat{\mathbf{q}}_{3}$, secular terms are removed from the equation (Strogatz, 1994) to obtain the following characteristic equations for $A=a \cdot e^{i \cdot \theta} / 2$

$$
O\left(\varepsilon^{2}\right): \quad \dot{a}=\hat{U} \cdot \beta_{1 r} \cdot a-\beta_{2 r} \cdot a^{3} \quad \dot{\theta}=\hat{U} \cdot \beta_{1 i}-\beta_{2 i} \cdot a^{2} .
$$

The complex-valued $\beta$ terms are computed as

$$
\begin{aligned}
& \beta_{1}=\beta_{1 r}+i \cdot \beta_{1 i}=\boldsymbol{Q}^{T} \cdot(\partial \boldsymbol{J} / \partial U) \cdot \boldsymbol{P}, \\
& \beta_{2}=\beta_{2 r}+i \cdot \beta_{2 i}=-\boldsymbol{Q}^{T} \cdot\left(2 \cdot \boldsymbol{C}\left(\boldsymbol{P}, \boldsymbol{Z}_{o}\right)+\boldsymbol{C}\left(\overline{\boldsymbol{P}}, \boldsymbol{Z}_{2}\right)+3 \cdot \boldsymbol{D}(\boldsymbol{P}, \boldsymbol{P}, \overline{\boldsymbol{P}}) / 4\right),
\end{aligned}
$$

where $\boldsymbol{Q}$ is the left eigenvector of the linear system: $\boldsymbol{Q}^{T} \cdot \boldsymbol{J}=i \cdot \omega^{*} \cdot \boldsymbol{Q}^{T}$, normalized such that $\boldsymbol{Q}^{T} \cdot \boldsymbol{P}=1$.

The steady-state solution of Eq. (22) provides the amplitude of the LCO

$$
a=\sqrt{\hat{U} \cdot \beta_{1 r} / \beta_{2 r}}
$$


If $\beta_{1 r}$ and $\beta_{2 r}$ have opposite signs, limit cycles will exist for negative values of $\hat{U}$ (i.e., $U<U^{*}$ ) which is a subcritical case (Fig. 1). Otherwise, a supercritical limit cycle exists. Furthermore, if $\beta_{1 r}$ is positive (which is the definition of a dynamically unstable flutter point (Ghommem et al., 2010), then the supercritical LCO will be very benign for large values of $\beta_{2 r}$. The complete solution is found by $\boldsymbol{q}=\boldsymbol{q}_{e}^{*}+\hat{\boldsymbol{q}}_{1}+\hat{\boldsymbol{q}}_{2}$ (having set $\varepsilon$ to unity). This can be done for a range of $\hat{U}$ values at little cost, once the nonlinear system parameters $\beta_{1}$ and $\beta_{2}$ are computed.

These direct tools are now used for the aeroelastic system described in the previous section (Fig. 4), with the change that, as noted above, gravitational effects (self-weight) have been removed from the system of equations, and the root angle $\alpha_{o}$ is fixed at $0^{\circ}$. Four cases are studied: both stall models in Fig. 3, with and without nonlinear beam modeling effects. The flutter velocity for each of these cases is $35.3 \mathrm{~m} / \mathrm{s}$, which is nearly identical to that found in Fig. 4. The results of Eqs. (23) and (24) are quantified in Table 1. From Eq. (23) it can be seen that $\beta_{1}$ is only dependent upon the flutter solution, and therefore would only be altered by nonlinearities implicitly. Because $\boldsymbol{q}_{e}^{*}$ has been set to zero for these computations, flutter is independent of the system nonlinearities, and so $\beta_{1}$ is as well. $\beta_{2}$, however, is dependent upon these nonlinearities as seen in the table, with the real part governing the amplitude of the LCO (Eq. 25), and the imaginary part governing the frequency.

Limit cycle behavior corresponding to these four cases is plotted in Fig. 5. Three analysis methods are utilized: timeintegration (as in Fig. 4), the method of multiple scales, and a spectral element method. This latter technique is a periodic monolithic time scheme which discretizes the cycle into elements, and uses a high-ordered polynomial as a temporal shape function. By fixing two degrees of freedom (typically the starting point and the amplitude of the LCO), the period $\left(\omega^{*}+\dot{\theta}\right)$ and velocity $\left(U^{*}+\hat{U}\right)$ may be computed along with the rest of the orbit. Additional details are provided by Beran et al. (2006). The main usefulness of the method in the current context is its ability to compute unstable subcritical LCOs, and thus ascertain the accuracy of MMS near the flutter point.

This is the situation encountered for case 1: the subcritical limit cycle is well-approximated by MMS up to roughly $1-2 \mathrm{~m} / \mathrm{s}$ below the flutter point. For this case, stalling nonlinearities exist only above an angle of attack of $10^{\circ}$ (Fig. 3), but because the perturbation is applied about $\boldsymbol{q}_{e}^{*}=0$ (which corresponds to $0^{\circ}$ angle of attack along the wing), the aerodynamics are fully linear from the vantage point of MMS. Clearly, for the wing parameters specified in conjunction with Fig. 2, the structural nonlinearities are destabilizing (softening). As $U$ is further decreased below $U^{*}$, portions of the wingtip begin to surpass the stalling angle of $10^{\circ}$ during the oscillation. The force decrement $\left(\Delta C_{z}, \mathrm{Eq} .10\right)$ is a stabilizing (hardening) nonlinearity, and so when enough of the wing has stalled, the limit cycle transitions from a low-amplitude unstable branch to a high-amplitude stable branch. The discontinuous $\Delta C_{z \alpha}$ stall curve precludes numerical convergence of the spectral element method within the deep-stall portion of the LCO (though Dunn and Dugundji, 1992; Tang and Dowell; 2002 show no such difficulty with the time-periodic harmonic balance method), but time-integration can be used to obtain this stable high-amplitude branch.

The cubic stalling curve is utilized in case 2, and now information pertaining to this aerodynamic nonlinearity is available to MMS (primarily via the cubic $\boldsymbol{D}(\boldsymbol{P}, \boldsymbol{P}, \overline{\boldsymbol{P}})$ of Eq. 24). The strong stabilizing effect of a cubic stall directly conflicts with the destabilizing structural nonlinearities (which are unchanged from case 1), resulting in a weakly subcritical limit cycle. This is correctly predicted by MMS, but the perturbation method has a very small range of accuracy, as higher-order stalling effects quickly transition the LCO to a stable high-amplitude branch. For cubic stall curves the spectral element method is convergent, and agrees well with the time-marching methods.

Cases 3 and 4 remove the nonlinear terms from the structural equations (Eqs. (1)-(3)). For a piecewise-linear stall curve, all nonlinearities have been removed from Eq. (24), $\beta_{2}$ is obviously zero, and the amplitude of the limit cycle $(a)$ is infinity i.e., the wing undergoes a pure unbounded flutter. For time marching, the oscillations grow until the wing stalls $\left(\alpha>10^{\circ}\right)$, and a limit cycle appears fully formed once the flutter speed is exceeded. This is typical for subcritical cases, of course, but here no LCOs exist for speeds beneath the flutter point. When cubic stall curves are used for case 4 , the expected supercritical behavior is observed. MMS is very accurate for this case, with errors in the LCO amplitude less than $4 \%$ at $5 \mathrm{~m} / \mathrm{s}$ above the flutter point (not shown in Fig. 5). This is an encouraging aspect of the method, as optimization studies (described below) will seek to obtain strongly supercritical limit cycles, thus improving both the aeroelastic behavior as well as the accuracy of the modeling tool.

To summarize, increasing $\beta_{2 r}$ (from left to right across Fig. 5) stabilizes the nonlinearities for supercritical behavior, decreasing the LCO amplitudes once the flutter speed has been exceeded. In order to explore this further, the strength of the limit cycle (as predicted by $\beta_{2 r}$ ) and the flutter speed is given in Fig. 6 as a function of the stiffness ratio $E I_{2} / E I_{1}$. This ratio is altered uniformly throughout the wing (as opposed to the optimization studies presented below, where different portions of the wing are allowed different mass/stiffness), and the dashed-line indicates the "baseline" design, which is the stiffness of the wing described in conjunction with Fig. 2, as well as the data in Fig. 5.

Strong softening geometric nonlinearities are evident for large stiffness ratios $(\sim 100)$, resulting in subcritical limit cycles. Recalling from Eqs. (1)-(3) that $E I_{2} / E I_{1}$ governs the structural nonlinearity; the problem becomes structurally linear when the ratio is unity. At this point, and for the piecewise-linear stall model used by case 1 , the entire aeroelastic perturbation is linear, and so $\beta_{2 r}$ becomes zero. Additional subcritical LCOs are seen for ratios below unity, but no modifications to the system of case 1 will result in supercritical behavior. The hardening effect of the cubic stall models (case 2) uniformly shift the previous curve in the positive $\beta_{2 r}$ direction, and supercritical LCOs exist for stiffness ratios slightly smaller than that used for the baseline wing. As with case 1 , the highest $\beta_{2 r}$ occurs at a ratio of unity where only aerodynamic nonlinearities exist. The limit cycles of case 4 (geometric nonlinearities turned off) show no dependence upon the stiffness ratio, as expected. 


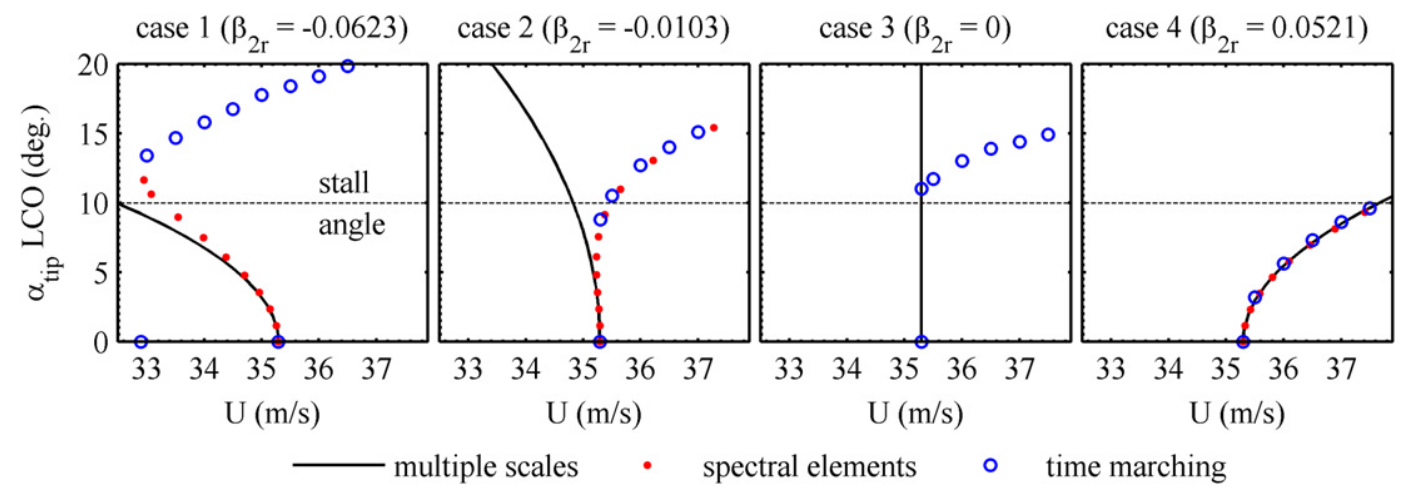

Fig. 5. Limit cycle behavior corresponding to the cases in Table 1.

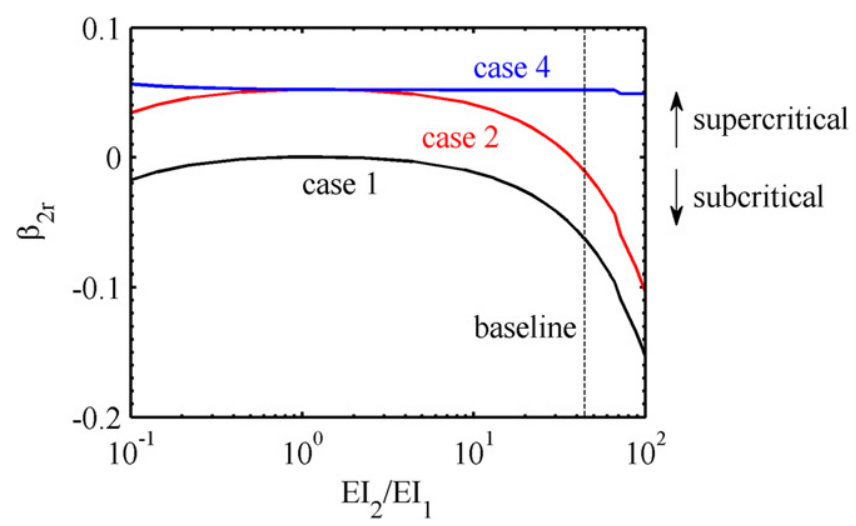

Fig. 6. Limit cycle behavior predicted by MMS for varying levels of structural nonlinearity.
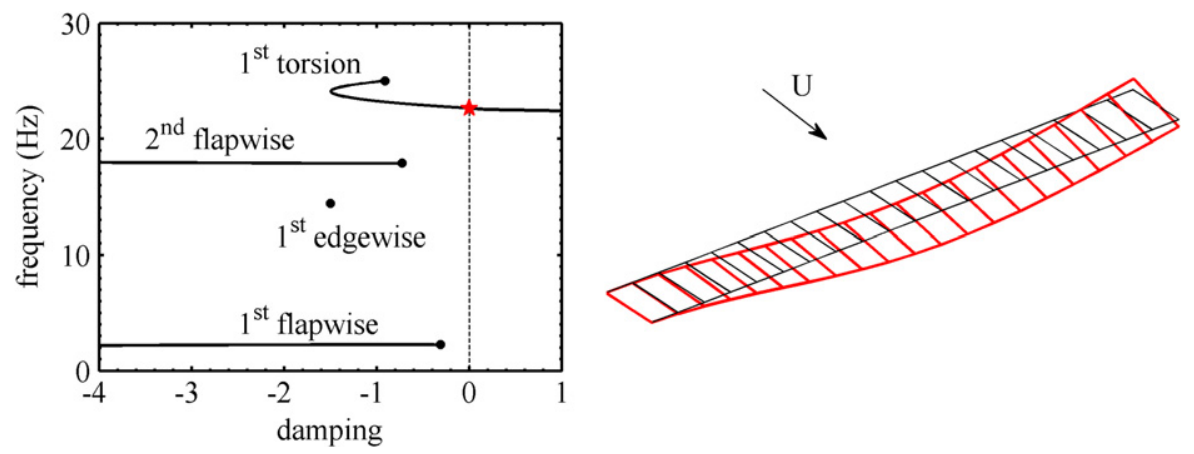

Fig. 7. Aeroelastic eigenvalue migration with increasing flight speed (left) and the mode shape $\boldsymbol{P}$ at flutter (right).

Table 1

$\beta$-parameters and LCO identification for various structural and aerodynamic nonlinearities.

\begin{tabular}{llllllll}
\hline Case & Stall model & Beam model & $\beta_{1 r}$ & $\beta_{1 i}$ & $\beta_{2 r}$ & $\beta_{2 i}$ & LCO \\
\hline 1 & Piecewise-linear & nonlinear & 0.2550 & -0.8113 & -0.0623 & 0.1146 & subcritical \\
2 & Piecewise-cubic & nonlinear & 0.2550 & -0.8113 & -0.0103 & -0.0523 & subcritical \\
3 & Piecewise-linear & linear & 0.2550 & -0.8113 & 0 & 0 & N/A \\
4 & Piecewise-cubic & linear & 0.2550 & -0.8113 & 0.0521 & -0.1669 & supercritical \\
\hline
\end{tabular}


Flutter occurs when the imaginary portions of the first torsional and second flapwise bending eigenvalues approach (with the former mode actually losing stability), as seen in Fig. 7. Changing the edgewise stiffness $E_{2}$ has no effect on this process, and so each data point in Fig. 6 has the same flutter speed, $35.3 \mathrm{~m} / \mathrm{s}$. This is not the case when the aspect ratio of the wing is altered, as seen in Fig. 8. A trade-off between linear and nonlinear performance is seen for low aspect ratios, where the flutter speed is large but the subsequent limit cycle is strongly subcritical. Flutter speed generally decreases with higher aspect ratios, and (as before), case 1 is unable to obtain supercritical behavior $\left(\beta_{2 r}>0\right)$. Supercritical limit cycles with cubic stall (case 2$)$ have an erratic and non-monotonic behavior with increasing aspect ratio. This would indicate the expectedly complex relationship between wing geometry and aerodynamic nonlinearities, but additional work is required to ascertain the extent to which this local behavior is sensitive to the numerous parameters used to define the stall model (Eqs. (7)-(10)).

Spatial variability in the inertial and elastic properties of the wing is now considered. Specifically, the derivative of the flutter speed and the $\beta$-terms (Eqs. (23) and (24)) are computed with respect to $E I_{1}, E I_{2}, G J, m$, and $I_{o}$ of each beam element (as defined in Eqs. (1)-(3)). For a given cross-section, these five terms cannot be controlled entirely separately (e.g., polynomial relationships typically exist between $E I_{1}$ and $m$ (Haftka and Gürdal, 1992), though advanced composite materials may provide a substantial level of diversity (Haddadpour and Zamani, 2012; Kameyama and Fukunaga, 2007). Specific relationships used to connect the five quantities for optimization will be discussed below; for this section their aeroelastic impact is considered separately. Gradients are computed with first-order finite differences, a technique whose accuracy is acceptable by virtue of the direct methods utilized above. Each derivative is further normalized for a logical comparison between stiffness and mass parameters (e.g., $\bar{m} \cdot\left(\partial U^{*} / \partial m\right)$, where $\bar{m}$ is the uniform baseline value of $0.235 \mathrm{~kg} / \mathrm{m}$ utilized above). Gradient results for the uniform wing are given in Fig. 9 along the span of the wing, for a beam structure divided into 20 finite elements. These results are computed under case 2 (Table 1) cubic stall and a nonlinear beam.

Concerning first the flutter derivatives, the general sign of each term reflects the idea that onset of flutter may be delayed by further separating the two modes which interact as stability is lost (first torsion and second flapwise bending, Fig. 7). As such, the $I_{o}$-gradients are negative and the GJ-gradients are positive, both of which would act to increase the uncoupled torsional frequency $\left(\pi \cdot \sqrt{G J / I_{o}} / 2 / L\right)$. Increasing $m$ and decreasing $E I_{1}$ would decrease the uncoupled second flapwise bending frequency $\left((\pi \cdot 1.49 / L)^{2} \cdot \sqrt{E I_{1} / m}\right)$. It should be understood that the frequency formulas given here will not directly equate to the eigenvalues in Fig. 7, which also include the inertia of the tip body (Fig. 1). Flutter derivatives with respect to stiffness are highest at the root (where beam stresses will be largest) and lowest at the tip (where stresses are zero). Derivatives with respect to inertia reflect the pertinent mode shape of Fig. 7, $I_{0}$-gradients follow the first torsion shape, and $m$-gradients the second flapwise bending shape. Flutter gradients with respect to $\mathrm{EI}_{2}$ are zero for the same reason as discussed above: edgewise bending does not participate in the loss of dynamic stability.

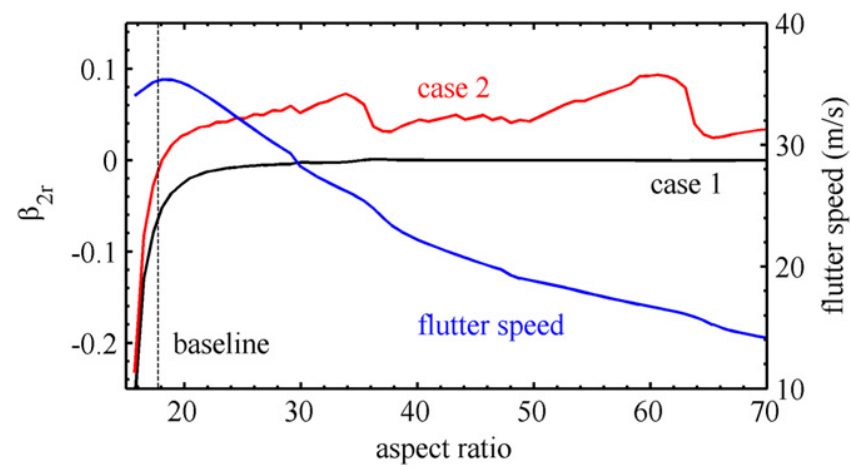

Fig. 8. Flutter and limit cycle behavior predicted by MMS as a function of wing aspect ratio.
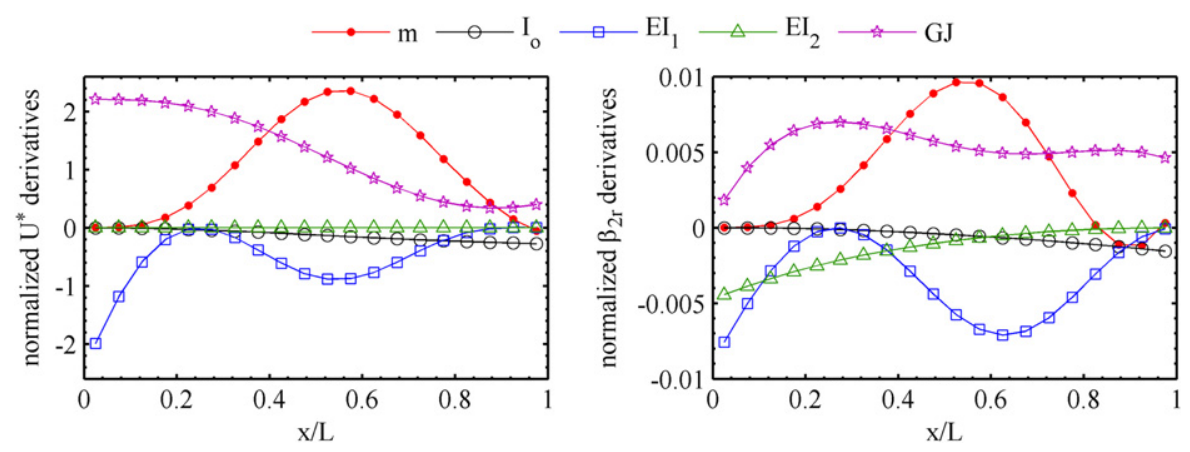

Fig. 9. Normalized derivatives of flutter speed (left) and limit cycles (right) with respect to spatial distribution of inertia and stiffness. 
Topologically, the $\beta_{2 r}$ derivatives on the right side of Fig. 9 share many similarities with the flutter gradients, though some differences are evident as well. Most importantly, $E I_{2}$-gradients are strongly negative at the root, which aligns with the trends in Fig. 6, decreasing the stiffness ratio weakens the destabilizing geometric nonlinearities, and increasing $\beta_{2 r}$ for supercritical LCOs. Similarly, one may expect positive $E I_{1}$-gradients, but like the flutter gradients, they remain negative. This would indicate that the implicit link between flutter and limit cycle is stronger than the explicit appearance of $E I_{1}$ in the nonlinear beam equations (Eqs. (1)-(3)). Torsional stiffness gradients are very dissimilar from the flutter gradients, though GJ does not appear as a nonlinear term. GJ will explicitly control the beam twist $\phi$, however, which has a nonlinear impact via the term $\left(\phi \cdot w_{, x x}\right)_{, x x}$. This explains the spatial trend of $\partial \beta_{2 r} / G J$, the first torsion mode (and hence $\phi$ ) is largest at the tip.

\section{Structural optimization}

Having obtained the flutter and limit cycle behavior in a direct manner, as well as the derivatives of this data with respect to the spatial distribution of stiffness and inertia (via finite differences), it is now desired to use this information to optimize the wing structure, accounting for both linear flutter and nonlinear post-flutter effects. Following Seyranian (1982), thickness- and width-based control functions are used to parameterize the wing structure/inertia. The vectors $\boldsymbol{h}_{\boldsymbol{w}}$ and $\boldsymbol{h}_{\boldsymbol{t}}$ represent these functions for each finite element

$$
\boldsymbol{h}_{\boldsymbol{w}}=\left\{\begin{array}{llll}
h_{w}^{1} & h_{w}^{2} & \cdots & h_{w}^{N-1}
\end{array}\right\}, \quad \boldsymbol{h}_{\boldsymbol{t}}=\left\{\begin{array}{llll}
h_{t}^{1} & h_{t}^{2} & \cdots & h_{t}^{N-1}
\end{array}\right\},
$$

where $N$ is the number of finite element nodes, and so $N-1$ is the number of finite elements. By using these functions to scale the width and thickness of each beam element (e.g., $t^{i}=\bar{t} \cdot \boldsymbol{h}_{\boldsymbol{t}}^{i}$, where $\bar{t}$ is a baseline thickness), and assuming that each cross-section is rectangular, the inertia/stiffness for the $i t h$ beam element is obtained as

$$
\begin{aligned}
& m^{i}=\bar{m} \cdot h_{t}^{i} \cdot h_{w}^{i}, \quad I_{o}^{i}=\bar{I}_{o} \cdot h_{t}^{i} \cdot\left(h_{w}^{i}\right)^{3}, \\
& E I_{1}^{i}=\overline{E I}_{1} \cdot h_{w}^{i} \cdot\left(h_{t}^{i}\right)^{3}, \quad E I_{2}^{i}=\overline{E I}_{2} \cdot h_{t}^{i} \cdot\left(h_{w}^{i}\right)^{3}, \quad G J^{i}=\overline{G J} \cdot h_{w}^{i} \cdot\left(h_{t}^{i}\right)^{3}
\end{aligned}
$$

The equations for $G J$ and $I_{o}$ further assume that the thickness of each cross section is much smaller than the width. Control values of unity keep the beam properties at the baseline values, which are indicated with an overbar.

The stiffness and inertial distributions along the beam, treated as separate in the previous section, have thus been linked under the assumption of a rectangular cross-section. This scenario lies closest to that utilized in the wind tunnel model of Tang and Dowell (2001), though flanges are also used to reduce torsional stiffness. Future work may consider different cross-sections (wing box (Butler and Banerjee, 1996), for example) or orthotropic materials with bendingextension-shear-twist coupling (Haddadpour and Zamani, 2012), in order to assess the impact of this structural parameterization on the optimization results.

The aeroelastic optimization problem considered here is formally written as

$$
\text { s.t. : }\left\{\begin{array}{l}
\max _{h_{\min }<h_{w}^{i}, h_{t}^{i}<h_{\max } \quad} \beta_{2 r} \\
U^{*} \geq \bar{U}^{*} \\
\sum m^{i} \leq \bar{m} \cdot(N-1)
\end{array} .\right.
$$

It is desired to maximize $\beta_{2 r}$ (which will provide low-amplitude supercritical limit cycles) by varying the control functions within each beam element. Side constraints $\left(h_{\min }, h_{\max }\right)$ are applied to each design variable, as well as a constraint that the flutter speed not decrease below that of the baseline design (which is the uniform wing studied above). The final constraint stipulates that the mass of the wing not exceed the mass of the baseline structure. All results are computed via case 2 (Table 1: cubic stall, nonlinear beam), gradients are computed using finite differencing (as in Fig. 9) and the method of moving asymptotes (Svanberg, 1987) is used for gradient-based optimization. Four optimization cases are run, each with different side constraints, as given in Table 2. Baseline data is also provided, with $\beta_{2 r}$ repeated from the data of Table 1.

Table 2

Aeroelastic optimization results.

\begin{tabular}{lrll}
\hline$h_{\min }, h_{\max }$ & \multicolumn{1}{l}{$\beta_{2 r}$} & $U^{*}(\mathrm{~m} / \mathrm{s})$ & $\sum m^{i} / \bar{m} /(N-1)$ \\
\hline 0 (baseline) & -0.0103 & 35.30 & 1 \\
\pm 0.05 & 0.0122 & 36.76 & 1 \\
\pm 0.10 & 0.0241 & 36.77 & 1 \\
\pm 0.15 & 0.0304 & 35.30 & 0.97 \\
\pm 0.20 & 0.0370 & 35.30 & 0.95 \\
\hline
\end{tabular}


As would be expected, increasing the size of the design space (via the side constraints) provides larger values of the objective function $\beta_{2 r}$, though the smallest bounds considered (0.05) are enough to transition the LCO behavior from subcritical to supercritical. The largest value obtained (0.0370) is smaller than the largest value in Fig. 6 (0.0521), presumably due to the fact that the current system is operating under a flutter constraint, and is unable to independently alter the edgewise and the flapwise bending stiffness. Between side constraints of 0.10 and 0.15 , the flutter constraint becomes active and the mass constraint inactive. The convergence history and optimal design for the case with side constraints of 0.20 can be seen in Fig. 10; the gradients of the optimal design with respect to stiffness and inertial parameters are given in Fig. 11.

As noted with regard to Fig. 9, the signs of the flutter and limit cycle gradients are largely the same for the baseline design. As such, the first few iterations in Fig. 10 improve both $U^{*}$ and $\beta_{2 r}$. The beneficial strategies as dictated by the baseline case (namely, frequency separation) gradually degrades the flutter speed however, which begins to decrease after the 7 th iteration, and ultimately converges to the constraint boundary $\overline{U^{*}}$. Presumably, this is due to a loss of stiffness at the root, which is seen in the right side of Fig. 10: both the thickness and the width control functions are at the lower bound. The gradients at the root (Fig. 11) highlight the flutter-limit cycle conflict, as the $E I_{1}$ - and the $G J$-gradients are each of opposite sign, and so any further improvement in $\beta_{2 r}$ is only obtained at the expense of $U^{*}$. As with the baseline case, $\mathrm{EI}_{2}$-gradients are prevalent in the limit cycle computations but have no role in the flutter speed, presenting further conflict at the wing root between the two metrics.

A second area of conflict is near the midspan $(x / L=0.6)$, where both control functions are again at the lower bound. In this area, the $m$ - and the $G J$-gradients are both of opposite sign: decreased interaction between torsional stiffness and translation inertia (of the second flapwise bending mode shape) would benefit the limit cycle oscillations, but degrade the flutter speed. The $E I_{1}$-gradients in this location, unlike at the root, have the same sign for both flutter and LCO. Outside of these two locations, the majority of the width variables are at the lower bound while most of the thickness variables are at the upper bound: the combined trend is towards a cross section with a smaller aspect ratio, decreasing the stiffness ratio and the strength of the softening nonlinearities, and providing strongly supercritical LCOs. It can also be seen that the magnitude of the $\beta_{2 r}$ gradients of the optimal design (Fig. 11) are much smaller than the baseline (Fig. 9), while the opposite is true for the flutter constraint.

Finally, the amplitudes of the limit cycle motion are given in Fig. 12 for both the baseline design (data repeated from the second plot of Fig. 5) as well as the optimal design of Fig. 10. As before, the perturbation data predicted by the method of multiple scales is compared to the spectral element method. Both designs have the same flutter point (the flutter constraint of Eq. (29) is active), but the subsequent limit cycles are very different, as expected: the baseline design is subcritical, the optimal design is supercritical. In terms of the angle of attack at the wing tip, the baseline design will
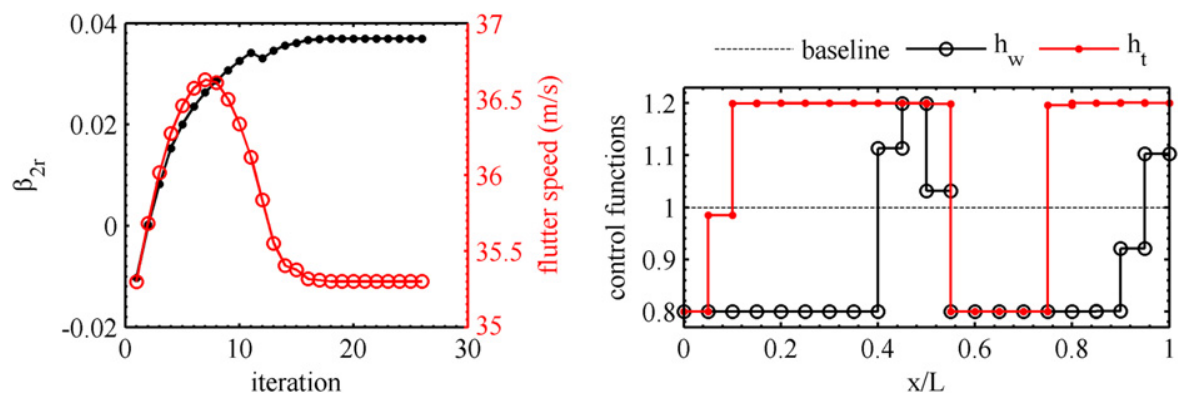

Fig. 10. Convergence history (left) and optimal control functions (right) with side constraints of \pm 0.2 .
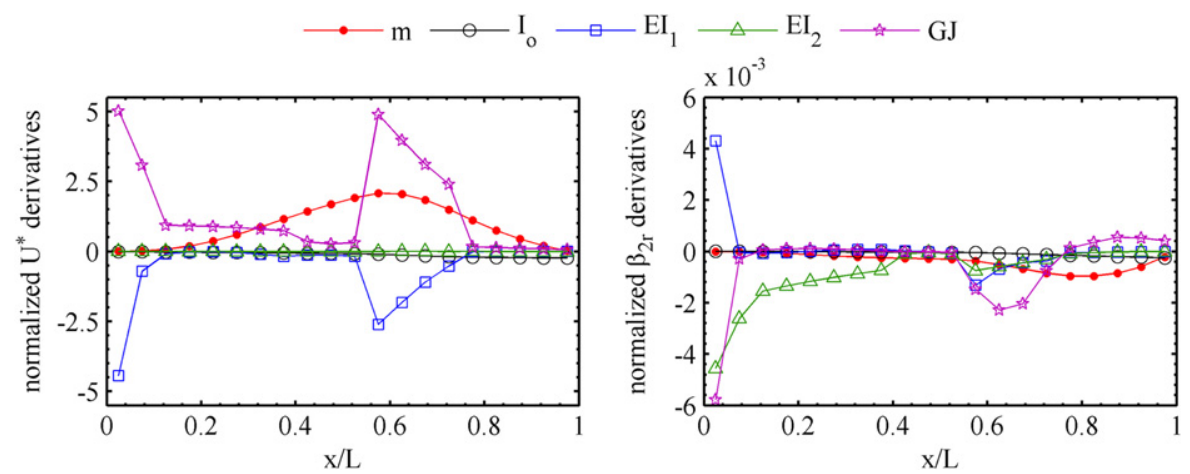

Fig. 11. Optimal design: normalized derivatives of flutter speed (left) and limit cycles (right). 

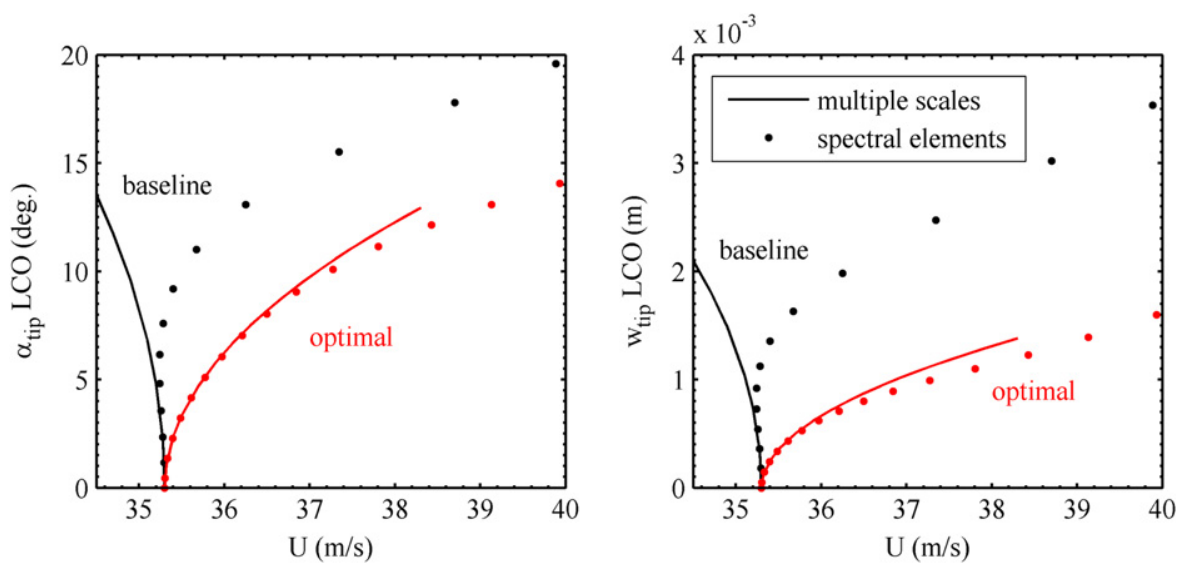

Fig. 12. Limit cycle behavior of the baseline and optimal designs.

suddenly jump to a high amplitude of $10^{\circ}$ once the flutter speed has been crossed, while the optimal deflections will remain very small.

This improved behavior in the immediate vicinity of the flutter point is the main goal and most important accomplishment of the optimization process. The specific design metrics (namely, $\beta_{2 r}$ ) only contain information pertaining to small nonlinear perturbations, and so a reduction of high amplitude behavior far removed from the flutter point cannot necessarily be expected from the optimal design. This improvement is present, however, with a twist amplitude $6^{\circ}$ less at a speed $5 \mathrm{~m} / \mathrm{s}$ above the flutter point. For the tip deflections (right side of Fig. 12), the reduction in limit cycle amplitude at this speed is even larger. As speculated above, it can now be confirmed that the optimization process has improved not only the character of the LCO, but the accuracy of the MMS process as well, which is reasonably accurate (6.5\% error in tip angle of attack) $3 \mathrm{~m} / \mathrm{s}$ above the flutter point for the optimal design. Indeed, the solution computed by MMS is conservative in the sense that the actual supercritical limit cycle (as computed with spectral elements) has a smaller amplitude than the perturbation solution.

\section{Conclusions}

This work has conducted the structural optimization of a highly flexible high-aspect-ratio wing first introduced by Tang and Dowell (2001). The nonlinear post-flutter aeroelasticity of this wing structure is dominated by destabilizing structural nonlinearities, and the subsequent subcritical limit cycle oscillation provides stable high-amplitude deflections at speeds well below the flutter point. This fact degrades the usefulness of a flutter speed constraint in the design optimization process, necessitating the inclusion of limit cycle metrics. Specifically, it is desired to design a wing structure with a benign low-amplitude supercritical LCO. To meet this requirement in an accurate and efficient manner, direct tools have been utilized to compute flutter and limit cycle behavior, the latter via the method of multiple scales. The resulting optimal design shows many interesting conflicts between flutter and LCO in terms of the preferred spatial distribution of inertia and stiffness, conflicts which did not exist for a uniform baseline wing.

Many future topics may be considered for the research effort outlined above. First, the effect of a non-zero trim state $\left(\boldsymbol{q}_{e}\right)$ may be considered, in order to assess its impact on the optimization process. Secondly, the structural parameterization of Eqs. (26)-(28) may be reconsidered. Superior optima (compared to that obtained in Fig. 12) are surely available when various structural and inertial terms can be considered in a more independent manner. This may be done via a higher level of geometric complexity at each cross section (Butler and Banerjee, 1996), or through the use of composite structures (Kameyama and Fukunaga, 2007). Third, the most suitable MMS metric for optimization should be considered. The current work uses $\beta_{2 r}$, but the amplitude of Eq. (25), some specific LCO amplitude (tip twist, for example) at a fixed flight speed, or some combination of these parameters may provide a superior structure. Finally, the effect of uncertainties needs to be accounted for in the design process, as LCO behavior has been shown to be susceptible to stochastic environments (Ghommem et al., 2010; Missoum et al., 2010; Sarkar et al., 2009; Stanford and Beran, 2012; Thomas et al., 2006). The direct method may be well suited to sampling-based techniques, as well as approximation schemes (first order reliability methods, e.g.) required when the number of uncertain parameters is large.

\section{Acknowledgments}

This work is sponsored by the Air Force Office of Scientific Research under Laboratory Task 03VA01COR (monitored by Dr. Fariba Fahroo). The authors are also grateful to Dr. J. Jaworski of the University of Cambridge and Dr. M. Ghommem of Virginia Tech for their technical insight concerning the tools used here. 


\section{References}

Badcock, K., Woodgate, M., 2010. Bifurcation prediction of large-order aeroelastic models. AIAA Journal 48, 1037-1046.

Badcock, K., Woodgate, M., Richards, B., 2004. Hopf bifurcation calculations for a symmetric airfoil in transonic flow. AIAA Journal 42, 883-892.

Beran, P., 1999. Computation of limit cycle oscillation using a direct method. In: Proceedings of the 40th AIAA Structures, Structural Dynamics, and Materials Conference, AIAA Paper No. 1999-1462.

Beran, P., Pettit, C., Millman, D., 2006. Uncertainty quantification of limit cycle oscillations. Journal of Computational Physics 217, $217-247$.

Bisplinghoff, R., Ashley, H., Halfman, R., 1955. Aeroelasticity. Addison-Wesley, Cambridge, MA.

Butler, R., Banerjee, J., 1996. Optimum design of bending-torsion coupled beams with frequency or aeroelastic constraints. Computers and Structures 60 , 715-724.

Chandiramani, N., Librescu, L., Plaut, R., 1996. Flutter of geometrically imperfect shear-deformable laminated flat panels using non-linear aerodynamics. Journal of Sound and Vibration 192, 79-100.

Cook, R., Malkus, D., Plesha, M., Witt, R., 2002. Concepts and Applications of Finite Element Analysis. Wiley, New York.

Cornwell, R., Malkus, D., 1992. Improved numerical dissipation for time integration algorithms in conduction heat transfer. Computer Methods in Applied Mechanics and Engineering 97, 149-156.

Demasi, L., Livne, E., 2009. Aeroelastic coupling of geometrically nonlinear structures and linear unsteady aerodynamics: two formulations. Journal of Fluids and Structures 25, 918-935.

Dowell, E., Edwards, J., Strganac, T., 2003. Nonlinear aeroelasticity. Journal of Aircraft 40, 857-874.

Dunn, P., Dugundji, J., 1992. Nonlinear stall flutter and divergence analysis of cantilevered graphite/epoxy wings. AIAA Journal 30, $153-162$.

Ghommem, M., Hajj, M., Nayfeh, A., 2010. Uncertainty analysis near bifurcation of an aeroelastic system. Journal of Sound and Vibration 329, $3335-3347$.

Gilliatt, H., Strganac, T., Kurdila, A., 2003. An investigation of internal resonance in aeroelastic systems. Nonlinear Dynamics 31, 1-22.

Griewank, A., Reddien, G., 1983. The calculation of Hopf points by a direct method. IMA Journal of Numerical Analysis 3, 295-303.

Haddadpour, H., Zamani, Z., 2012. Curvilinear fiber optimization tools for aeroelastic design of composite wings. Journal of Fluids and Structures 33, $180-190$

Haftka, R., 1986. Structural optimization with aeroelastic constraints: a survey of US applications. International Journal of Vehicle Design 7, 381-392.

Haftka, R., Gürdal, Z., 1992. Elements of Structural Optimization. Kluwer Academic Publishers, Dordrecht, The Netherlands.

Hodges, D., Dowell, E., 1974. Nonlinear equations of motion for the elastic bending and torsion of twisted nonuniform rotor blades. NASA TN D, 7818.

Kameyama, M., Fukunaga, H., 2007. Optimum design of composite plate wings for aeroelastic characteristics using lamination parameters. Computers and Structures 85, 213-224.

Librescu, L., Marzocca, P., Silva, W., 2002. Supersonic/hypersonic flutter and postflutter of geometrically imperfect circular cylindrical panels. Journal of Spacecraft and Rockets 39, 802-812.

Missoum, S., Dribusch, C., Beran, P., 2010. Reliability-based design optimization of nonlinear aeroelasticity problems. Journal of Aircraft 47, 992-998.

Morton, S., Beran, P., 1999. Hopf-bifurcation analysis of airfoil flutter at transonic speeds. Journal of Aircraft 36, 421-429.

Nayfeh, A., Balachandran, B., 1995. Applied Nonlinear Dynamics. John Wiley and Sons, New York.

Nayfeh, A., Ghommem, M., Hajj, M., 2012. Normal form representation of the aeroelastic response of the Goland wing. Nonlinear Dynamics 76, $1847-1861$.

Nichkawde, C., Strganac, T., Beran, P., 2006. Nonlinear aeroelastic response of the flexible wing in trim. In: Proceedings of the 47th AIAA Structures, Structural Dynamics, and Materials Conference, AIAA Paper No. 2006-1637.

Palacios, R., Murua, J., Cook, R., 2010. Structural and aerodynamic models in nonlinear flight dynamics of very flexible aircraft. AIAA Journal 48, 2648-2659.

Palaniappan, K., Beran, P., Jameson, A., 2006. Optimal control of LCOs in aero-structural systems. In: Proceedings of the 47th AIAA Structures, Structural Dynamics, and Materials Conference, AIAA Paper No. 2006-1621.

Paolone, A., Vasta, M., Luongo, A., 2006. Flexural-torsional bifurcations of a cantilever beam under potential and circulatory forces II post-critical analysis. International Journal of Non-Linear Mechanics 41, 595-604.

Patil, M., Hodges, D., 2004. On the importance of aerodynamic and structural geometrical nonlinearities in aeroelastic behavior of high-aspect-ratio wings. Journal of Fluids and Structures 19, 905-915.

Patil, M., Hodges, D., Cesnik, C., 2001. Limit cycle oscillations in high-aspect-ratio wings. Journal of Fluids and Structures 15, 107-132.

Peters, D., 1985. Toward a unified lift model for use in rotor blade stability analysis. Journal of the American Helicopter Society 30, 32-42.

Sarkar, A., Witteveen, J., Loeven, A., Bijl, H., 2009. Effect of uncertainty on the bifurcation behavior of pitching airfoil stall flutter. Journal of Fluids and Structures $15,304-320$.

Seyranian, A., 1982. Sensitivity analysis and optimization of aeroelastic stability. International Journal of Solids and Structures 18, $791-807$.

Shearer, C., Cesnik, C., 2007. Nonlinear flight dynamics of very flexible aircraft. Journal of Aircraft 44, 1528-1545.

Stanford, B., Beran, P., 2011. Optimal structural topology of a plate-like wing for subsonic aeroelastic stability. Journal of Aircraft 48, 1193-1203.

Stanford, B., Beran, P., 2012. Computational strategies for reliability-based structural optimization of aeroelastic limit cycle oscillations. Structural and Multidisciplinary Optimization 45, 83-99.

Stanford, B., Beran, P., Kurdi, M., 2010. Adjoint sensitivities of time-periodic nonlinear structural dynamics. Computers and Structures 88, 1110-1123.

Strogatz, S., 1994. Nonlinear Dynamics and Chaos. Perseus Books, Reading, MA.

Svanberg, K., 1987. The method of moving asymptotes - a new method for structural optimization. International Journal for Numerical Methods in Engineering 24, 359-373.

Tang, D., Dowell, E., 2001. Experimental and theoretical study on aeroelastic response of high-aspect-ratio wings. AIAA Journal 39, $1430-1441$.

Tang, D., Dowell, E., 2002. Limit-cycle hysteresis response for a high-aspect-ratio wing model. Journal of Aircraft 39, 885-888.

Thomas, J., Dowell, E., Hall, K., 2004. Modeling viscous transonic limit-cycle oscillation behavior using a harmonic balance approach. Journal of Aircraft 41, 1266-1274.

Thomas, J., Dowell, E., Hall, K., Denegri, C., 2006. An investigation of the sensitivity of F-16 fighter flutter onset and limit cycle oscillations to uncertainties. In: Proceedings of the 47th AIAA Structures, Structural Dynamics, and Materials Conference, AIAA Paper No. 2006-1847.

Tran, C., Petot, D., 1981. Semi-empirical model for the dynamic stall of airfoils in view to the application to the calculation of responses of a helicopter blade in forward flight. Vertica 5, 35-53.

Wang, Z., Chen, P., Liu, D., Mook, D., 2010. Nonlinear-aerodynamics/nonlinear-structure interaction methodology for a high-altitude long-endurance wing. Journal of Aircraft 47, 556-566.

Woodgate, M., Badcock, K., 2007. Fast prediction of transonic aeroelastic stability and limit cycles. AIAA Journal 45, $1370-1381$. 